\title{
EDITORIAL
}

\section{COVID-19: Reasons for Guarded Optimism}

\author{
Noel Snell ${ }^{1}$ \\ Published online: 16 February 2021 \\ (c) The Author(s), under exclusive licence to Springer Nature Switzerland AG part of Springer Nature 2021
}

Coronaviruses have been known to be potentially pathogenic for many years; four subtypes cause about $30 \%$ of 'common colds' in man, and many others cause infections of varying severity in animals. Because they generally caused mild disease, little research was done into specific anti-coronavirus agents until recently (I co-authored one of the early studies of a potential anti-coronavirus therapy in 1991-it did not work) [1], although effective vaccines have been developed for several commercially important animal coronavirus infections [2]. The situation changed in 2002, when a novel coronavirus caused severe and often fatal disease in Hong Kong and spread to several other countries, notably Canada. Named 'Severe Acute Respiratory Syndrome' or SARS, this epidemic luckily was contained and disappeared fairly swiftly; but in 2012 another novel and frequently fatal coronavirus infection occurred in the Middle East where fortunately it remained localised. Named MERS (Middle East Respiratory Syndrome), sporadic cases still occur [3].

Despite these warnings, and pressure from the scientific community, governments and funding agencies relaxed, and little research continued to be done into vaccines or specific treatments against these potentially deadly agents. As a result, when the new pandemic arrived, caused by the rapidly named SARS-CoV-2 virus (the disease it causes being called COVID-19), the world was woefully unprepared.

SARS was more deadly than COVID-19, and symptoms appeared at the time it became infectious; both factors made it easier to identify and contain. SARS-CoV-2 is less deadly, but more infectious, and can be transmitted days before symptoms appear (and many cases remain asymptomatic), an ideal combination promoting transmission and leading to the current pandemic situation. Mistakes were made in dealing with the disease early on, from the use of existing antimicrobials that turned out to be ineffective (lopinavir/ritonavir)

Noel Snell

nsnell@ic.ac.uk

1 National Heart and Lung Institute, Imperial College London, London, UK and in some cases also potentially harmful (hydroxychloroquine, azithromycin), to delays in some countries (including the UK) in setting up an effective 'test and trace' system. Discharging untested elderly patients from hospitals into care homes led to large numbers of deaths in the UK. As hospitals filled up and mortality rose, travel restrictions were imposed in many countries, and shops, restaurants, public amenities, places of entertainment, and schools were closed in an effort to contain the infection, at enormous global economic cost. As the first wave of infection came under control authorities relaxed these restrictions, and encouraged the return of children and young adults to schools and universities. As it had been shown in 2006 that the Autumn return to school in Canada triggers a large increase in virally induced asthma in school children about 2 weeks later, and subsequently a similar increase in adult family members at home after another week [4], it was predictable that a similar pattern of increased transmission might be seen with SARSCoV-2. The world is now experiencing a second wave of infection, made worse by the emergence of more infectious variant viruses in the UK and South Africa.

So why are there grounds for optimism? First, the extraordinary achievement of the scientific community and pharmaceutical industry in developing new vaccine candidates from scratch in under a year. At the time of writing (January 2021) several groups have released promising results on their vaccines. The Pfizer/BioNTech [5] and Moderna/NIH [6] vaccines both seem to be about 90-95\% effective; Russia's Sputnik $\mathrm{V}$ vaccine claims an efficacy of $91.4 \%$, based on interim analysis of data obtained 7 days after the second dose of vaccine. The Oxford University/AstraZeneca findings are more complicated but at worst indicate $62 \%$ effectiveness, apparently greater with some sub-groups of subjects and with different vaccination schedules [7]. No major safety issues have been identified with any of the candidates to date, although a few cases of severe allergic reaction have been reported, possibly related to polyethylene glycol included in some of the vaccines [8]. The Oxford/AstraZeneca vaccine has the benefits that it is relatively inexpensive and can be stored in a standard domestic refrigerator (the other current main 
candidates require ultracold storage), which could make it particularly valuable for use in less developed countries. At the time of writing, approval processes (often under emergency authorisation procedures) are underway worldwide; for example, the Pfizer/BioNTech and Moderna/NIH vaccines are now available in the USA, and the European Medicines Agency has recommended approval of the Pfizer/ BioNTech vaccine and are currently reviewing the Moderna/ NIH vaccine. The Pfizer/BioNTech, Moderna, and Oxford/ AstraZeneca vaccines have recently been approved for use by the UK regulatory authority, so effective vaccines are now available to us with the potential to protect the vulnerable and to interrupt the cycle of infection, with the prospect of life beginning to return to something more like normality.

A second reason for optimism is that mortality in hospitalised patients during this second wave of infection seems to be lower than during the first wave [9]. In part, this may be because, sadly, many of the most vulnerable have already perished in the first wave. However, much of the improvement is due to better treatment; clinicians have learned the best ways to manage oxygenation and ventilation, and now have at least one proven therapy-the use of the inexpensive steroid dexamethasone in more seriously ill patients has been shown in the UK RECOVERY study to reduce mortality by up to one-third [10]. After initial hype, and emergency authorisation in several countries for use in hospitalised patients, the antiviral remdesivir seems to confer only marginal benefits in shortening symptom duration, and has no proven effect on mortality [11]. The Janus Kinase inhibitor, baricitinib, when added to remdesivir, reduced recovery time and improved clinical status in patients receiving high-flow oxygen or noninvasive ventilation, compared with those on remdesivir alone [12]. Other potential therapies are being evaluated; currently, inhaled interferon-beta, developed by a small UK company, Synairgen, looks interesting, with hospitalised patients given the active drug having greater odds of improvement and a more rapid recovery than those on placebo [13]. Several companies are developing monoclonal antibodies to the coronavirus spike protein, the best-known preparation being Regeneron's REGN-COV2 (as administered to US President Trump) [14]. So far, the antibodies studied seem safe and have early evidence of efficacy. The monoclonal antibody bamlanivimab, has recently been given emergency use authorisation by the US Food and Drug Administration (FDA) for treating mild-to-moderate COVID-19 in non-hospitalised adults and children [15], and in November AstraZeneca started late-stage trials of a longacting monoclonal antibody combination drug (AZD7442) to prevent COVID-19 infection in at-risk people. However, treatment with convalescent plasma (which should be rich in natural antibodies) has failed to prevent severe disease or death in controlled studies [16, 17], although a very recent study showed a decrease in progression to severe disease when high-titre convalescent plasma was given within 72 $\mathrm{h}$ after the onset of mild symptoms [18]. Because severely ill patients may exhibit hyper-reactivity of their immune response ('cytokine storm'), antagonists of the mediator interleukin-6 have been given in open-label and clinical trials [19]. One (Roche's tocilizumab) has given mixed results; a recent study showed benefit on a combined clinical endpoint when given to critically ill patients [20], and more detailed results from this study (currently awaiting peer review) demonstrate improved survival with both tocilizumab and another IL-6 inhibitor, sarilumab. Many other potential treatments are in clinical trials, often existing medications which are being repurposed (the benefit being that they are already approved for other conditions, have a known safety profile, are readily available, and often inexpensive) [21]. Glycyrrhizin was shown to be highly active in vitro against SARS-CoV [22] but does not seem to have been tested against SARS-CoV2. Severe COVID-19 is characterised by hypercoagulability of the blood, with a pathological picture which can resemble the rare condition known as antiphospholipid syndrome, which responds to aspirin; this is now under study in COVID-19. Vitamins $C$ and D have both been studied clinically in COVID-19 for their immunostimulant effects, but neither have been proven to modify the disease $[23,24]$. An interesting observation is that SARS$\mathrm{CoV}-2$ infections seem less frequent in countries which have a routine vaccination programme against tuberculosis (caused by Mycobacterium tuberculosis) using the BCG vaccine. This is known to have non-specific immunostimulant effects, but a recent study has found that there are strong similarities between a SARS-CoV-2 envelope protein and a protein unique to mycobacteria, raising the possibility that BCG vaccine could generate a degree of specific immunity to the virus [25].

Particularly in regard to vaccines, there are currently good grounds for (guarded) optimism. However, there are still many challenges ahead; to control the pandemic probably $60-70 \%$ of the global population will need to become immune, either as a result of natural infection, or vaccination. 'Vaccine deniers' in some countries (e.g., the USA) may make this target difficult to achieve. Vaccinating twothirds of the world's population will be a challenging and lengthy process [26]. It is also becoming clear that many patients who recover from COVID-19-even those with mild disease-may experience a variety of symptoms (socalled 'long Covid') including fatigue, muscle aches, and difficulty concentrating, for weeks or months afterwards; and $18 \%$ of patients in the USA have been reported to receive a psychiatric diagnosis within 14-90 days of their illness [27], so lengthy rehabilitation programmes may need to be established [28]. Let us hope that governments learn the lesson from this viral pandemic and start now to prepare for the next one-which may not be caused by another 
coronavirus, but is very likely to originate in animals, possibly (like SARS, Ebola, and COVID-19), in bats [29]. At the very least, the World Health Organization's global surveillance programmes should have guaranteed funding, and an international programme of research into both specific anticoronavirus agents and broad-spectrum antivirals should be established.

\section{Declarations}

Funding No funding was received for the publication of this editorial.

Conflicts of interest Dr Noel Snell FRCP FFPM FRSB is an honorary clinical senior lecturer at the National Heart and Lung Institute, Imperial College London, and former director of research at the British Lung Foundation. He has a particular interest in respiratory infections. He has worked for AstraZeneca in the past but is not a shareholder.

Ethics approval Not applicable.

Consent to participate Not applicable.

Consent for publication Not applicable.

Availability of data and material Not applicable.

Code availability Not applicable.

Authors' contributions Noel Snell researched and wrote this editorial.

\section{References}

1. Higgins P, Barrow G, Tyrrell D, et al. A study of the efficacy of the immunomodulatory compound 7-thia-8-oxguanosine in coronavirus 229E infections in human volunteers. Antiviral Chem Chemother. 1991;2:61-4.

2. Tizard I. Vaccination against coronaviruses in domestic animals. Vaccine. 2020;38:5123-30. https://doi.org/10.1016/j.vacci ne.2020.06.026.

3. Weiss S. Forty years with coronaviruses. J Exp Med. 2020;217:e20200537.

4. Johnston N, Johnston S, Norman G, et al. The September epidemic of asthma hospitalization: school children as disease vectors. J Allergy Clin Immunol. 2006;117:557-62.

5. Polack F, Thomas S, Kitchin N, et al. Safety and efficacy of the BNT162b2 mRNA Covid-19 vaccine. N Engl J Med. 2020;383:2603-15. https://doi.org/10.1056/NEJMoa2034577.

6. Baden L, El-Sahly H, Essink B, et al. Efficacy and safety of the mRNA-1273 SARS-CoV-2 vaccine. N Engl J Med. 2020. https:// doi.org/10.1056/NEJMoa2035389.

7. Voysey M, Clemens S, Madhi S, et al. Safety and efficacy of the ChAdOx1nCoV-19 vaccine (AZD1222) against SARS-CoV-2: an interim analysis of four randomised controlled trials in Brazil, South Africa, and the UK. Lancet. 2020. https://doi.org/10.1016/ S0140-6736(20)32661-1.

8. de Vrieze J. Pfizer's vaccine raises allergy concerns. Science. 2021;371:10-1.

9. Ledford $\mathrm{H}$. Why do COVID death rates appear to be falling? Nature. 2020;587:190-2.
10. The RECOVERY Collaborative Group. Dexamethasone in hospitalized patients with COVID-19-preliminary report. N Engl J Med. 2020. https://doi.org/10.1056/NEJMoa2021436.

11. Davies M, Osborne V, Lane S, et al. Remdesivir in treatment of COVID-19: a systematic benefit-risk assessment. Drug Saf. 2020;43:645-56.

12. Kalil A, Patterson T, Mehta A, et al. Baricitinib plus remdesivir for hospitalized adults with Covid-19. N Engl J Med. 2020. https ://doi.org/10.1056/NEJMoa2031994.

13. Monk P, Marsden R, Tear V, et al. Safety and efficacy of inhaled nebulised interferon beta-1a (SNG001) for treatment of SARSCoV-2 infection: a randomised, double-blind, placebo-controlled, phase 2 trial. Lancet Respir Med. 2020. https://doi.org/10.1016/ S2213-2600(20)30511-7.

14. DeFrancesco L. COVID-19 antibodies on trial. Nature Biotechnol. 2020;38:1242-52. https://doi.org/10.1038/s41587-020-0732-8.

15. Mahase E. Covid-19: FDA authorises neutralising antibody bamlanivimab for non-admitted patients. BMJ. 2020;371:m4362.

16. Agarwal A, Mukherjee A, Kumar G, et al. Convalescent plasma in the management of moderate COVID-19 in adults in India: open label phase II multicentre randomised controlled trial (PLACID Trial). BMJ. 2020;371:m3939.

17. Simonovich V, Burgos Pratx L, Scibona P. A randomized trial of convalescent plasma in COVID-19 severe pneumonia. N Engl J Med. 2020. https://doi.org/10.1056/NEJMoa2031304.

18. Libster R, Perez Marc G, Wappner D, et al. Early high-titer plasma therapy to prevent severe COVID-19 in older adults. N Engl J Med. 2021. https://doi.org/10.1056/NEJMoa2033700.

19. Zhou Z, Price C. Overview on the use of IL- 6 agents in the treatment of patients with cytokine release syndrome (CRS) and pneumonitis related to COVID-19 disease. Expert Opin Invest Drugs. 2020;29:1407-12. https://doi.org/10.1080/13543784.2020.18405 49.

20. Wise J. COVID-19: critically ill patients treated with arthritis drug tocilizumab show improved outcomes, researchers report. BMJ. 2020;371:m4530.

21. Gordon D, Jang G, Bouhaddou M, et al. A SARS-CoV-2 protein interaction map reveals targets for drug repurposing. Nature. 2020;583:459-68. https://doi.org/10.1038/s41586-020-2286-9.

22. Cinati J, Morgenstern B, Bauer G, et al. Glycyrrhizin, an active component of liquorice roots, and replication of SARS-associated coronavirus. Lancet. 2003;361:2045-6.

23. Holford P, Carr C, Jovic T, et al. Vitamin C - an adjunctive therapy for respiratory infection, sepsis and COVID-19. Nutrients. 2020;12:3760. https://doi.org/10.3390/nu12123760.

24. Murai I, Fernandes A, Sales L et al. Effect of Vitamin D3 supplementation vs placebo on hospital length of stay in patients with severe COVID-19: a multicenter, double-blind, randomized controlled trial. medRxiv. https://bit.ly/3mfqOeH

25. Nuovo G, Tili E, Suster D, et al. Strong homology between SARSCoV-2 envelope protein and a Mycobacterium sp. antigen allows rapid diagnosis of mycobacterial infections and may provide specific anti-SARS-CoV-2 immunity via the BCG vaccine. Ann DiagnPathol. 2020;48:151600.

26. Arnold C. The biggest logistics challenge in history. New Sci. 2020;3309:36-40.

27. Taquet M, Luciano S, Geddes J, Harrison P. Bidirectional associations between COVID-19 and psychiatric disorder: retrospective cohort studies of 62354 COVID-19 cases in the USA. Lancet Psychiatry. 2020. https://doi.org/10.1016/S2215-0366(20)30462 -4 .

28. Wade D. Rehabilitation after COVID-19: an evidence-based approach. Clin Med. 2020;20:359-64.

29. Snell N. Bats and human health. Biologist. 2008;55:72-4. 